\title{
Integración y diferenciación cooperativa: de las secciones a los grupos de sociedades
}

\author{
Carlos Vargas Vasserot \\ Profesor Titular Derecho Mercantil (Universidad de Almería)
}

Recibido: $\quad 20.06 .10$

Aceptado: 02.07 .10

Sumario: 1. Introducción. 2. Los distintos niveles de la intercooperación económica. 3. Integración y diferenciación cooperativa. 4. Los grupos cooperativos y las limitaciones legales para la constitución de filiales. 5. Algunas experiencias empresariales para superar las limitaciones financieras que presenta el régimen de las sociedades cooperativas. 6. Conclusiones. 7. Bibliografía.

Resumen: Las cooperativas se han querido enfrentar a los desafíos globales del mercado a través de estrategias de crecimiento. El presente estudio pretende poner de relieve como presiona la legislación cooperativa, incentivada por políticas públicas, para la creación de grupos cooperativos y cooperativas de segundo grado. Con una visión crítica se analizan las disposiciones legales que incentivan, la mayoría de veces sin éxito, estos mecanismos de agrupación e integración cooperativa. A su vez se estudia el fenómeno contrario de disgregación, con el estudio del fenómeno de las secciones de cooperativas contraponiéndolo a la creación de filiales típica de las sociedades de capital. El análisis de casos reales en España y en varios países de nuestro entorno económico, ponen de manifiesto diversos recursos para aplicar con éxito políticas de expansión empresarial.

Palabras clave: Integración, cooperativas de segundo grado, colaboración empresarial.

Abstract: In confronting global market challenges, cooperatives have often focused on strategies of growth all the while preoccupied about the degree to which cooperatives principles can be maintained. This paper illustrates cooperative legislation's role in shaping a legal environment in which cooperatives can both utilize necessary competitive strategies and maintain cooperative principles. Given the recent global financial crisis and the resulting lack of confidence in the shareholder model as the overriding paradigm, this task is even more relevant. We are critical of certain legislative provisions which do not achieve their stated goals and hide economic realities, encouraging cooperatives to "engineer» around such legislation or, alternatively, utilize cooperative legal mechanisms to find «refuge». Notwithstanding the apparent «pro» cooperative legal environment in Spain, in particular, its emphasis on integra- 
tion, we argue that the legislation is inadequate: Strategies of integration are often utilized not out of the necessity to pursue a justified economic growth strategy but simply to allow for the subsistence of small cooperatives. In addition, such integration strategies overlook the need for flexibility and differentiation (i.e. mechanisms within cooperative administrations to deal with particularities and distinct objectives). In the case of cooperatives with justified growth needs, the legislation creates limitations for development and expansion within the cooperative framework, thus encouraging resort to ingenious methods to dodge such limitations or change form to an investor owned firm. I argue for a legislative regime that acknowledges the necessity of both growth and differentiation, allowing flexibility so that cooperatives of varying size can continue in a socially valuable framework. I refer to other legislative mechanisms from selected countries as examples of what can be done to complement and make coherent the stated intent of the Spanish legislation and the actual results. ship.

Key words: Integration, second degree cooperatives, business partner- 


\section{Introducción}

La búsqueda de la óptima dimensión empresarial ha constituido siempre una preocupación del sector cooperativo que ve en los procesos de concentración empresarial la forma, si no la única, sí la mejor de afrontar los retos de la globalización, y a la vez de superar algunas de las limitaciones estructurales y económicas que tienen las sociedades cooperativas para su expansión y crecimiento sin tener que abandonar dicho modelo empresarial ${ }^{1}$. Este agrupamiento empresarial es incentivado por los poderes públicos a través de la adopción de diversas medidas tendentes a favorecer las estructuras de integración económica de las cooperativas.

Así en España lo reconoce la Ley 27/1999 estatal de Cooperativas (se cita LCoop) en varios momentos de su Exposición de Motivos («Son de especial interés las formas de colaboración económica entre cooperativas, procurando su ampliación y facilitando la integración»; "Las especiales características de las sociedades cooperativas han hecho necesaria la regulación del grupo cooperativo, con la finalidad de impulsar la integración empresarial de este tipo de sociedades, ante el reto de tener que operar en mercados cada vez más globalizados») y, en particular, en el artículo 108.1 («Se reconoce como tarea de interés general, a través de esta Ley y de sus normas de aplicación, la promoción, estímulo y desarrollo de las sociedades cooperativas y de sus estructuras de integración económica y representativa»).

Este fomento de las relaciones intercooperativas muchas veces se han traducido en subvenciones, desgravaciones o créditos preferentes, sin contar con el tratamiento como resultados cooperativos de los ingresos derivados de participaciones en sociedades cuando éstas realicen actividades preparatorias, complementarias o subordinadas a las de la propia cooperativa [art. 57.3, letra a) LCoop]; y la calificación de actividades cooperativizadas a las operaciones de suministro y entrega de productos o servicios realizadas entre cooperativas (art. 79.3 LCOOP). ${ }^{2}$

1 Sobre estos aspectos: Barea/Juliá/Monzón, 1999, passim; Chaves/Fajardo/Namorado, 2003, passim.

2 A efectos fiscales, además de la consideración de ingresos cooperativos de los intereses y retornos procedentes de la participación de la cooperativa en otra cooperativa (art. 17.5 Ley de Régimen Fiscal de Cooperativas), hay que reseñar la neutralidad fiscal con la que tributan gran parte de las modalidades de reestructuración societaria, extensibles a las cooperativas (arts. 83-96 Ley del Impuesto de Sociedades, y más recientemente la Ley 25/2006 extiende el régimen fiscal de las reorganizaciones empresariales a las Sociedades Cooperativas Europeas que cambien de domicilio). 


\section{Los distintos niveles de la intercooperación económica}

La fenomenología que cabe en un concepto amplio de integración empresarial, y que responde a la faceta puramente económica del «principio cooperativo de intercooperación» (el sexto de la lista de la $\mathrm{ACl}$ de 1995), es muy variada ${ }^{3}$. De más a menos en cuanto a los niveles de unión empresarial que consiguen los sujetos que participan en los procesos de concentración empresarial, y fijándonos en la legislación española de cooperativas, tenemos en primer lugar las fusiones (arts. 63-67 LCoop), operación societaria que supone creación de una nueva cooperativa por la unión jurídica de dos o más sociedades, o la absorción por una cooperativa de una o varias sociedades ya existentes que desaparecen.

En un escalafón menor en esta escala del nivel de cohesión alcanzado por las sociedades que forman parte de los procesos de concentración, al no suponer una modificación estructural ni la unificación jurídica total de las empresas participes, tenemos las modalidades de integración cooperativa en sentido estricto, en particular, las cooperativas de segundo grado y los grupos cooperativos.

El término integración tiene en el cooperativismo entidad propia frente al de concentración empresarial con el que se denominan las parecidas técnicas que utilizan las sociedades de capital para crear empresas de gran tamaño. Se quiere así subrayar, de un lado, que las cooperativas se integran pero conservan su individualidad y, de otro, que estas realidades grupales tienen en Derecho cooperativo particularidades jurídicas y económicas propias ${ }^{4}$. Si la fusión supone una total integración, económica y jurídica de las entidades que participan en ella (concentración en la unidad), la integración cooperativa mantienen las unidades que se agrupan (concentración en la pluralidad) 5 .

Las cooperativas de segundo grado (art. 77 LCoop) constituyen el mecanismo por excelencia de integración cooperativa, con una indivi-

Anótese que todos los años se convocan subvenciones destinadas al fomento de la integración cooperativa tanto en el ámbito estatal (Orden APA/180/2008) como en el autonómico (por poner un solo ejemplo, en Navarra Orden 77/2008 de la Consejera de Desarrollo Rural y Medio Ambiente), para sufragar total o parcialmente los estudios de viabilidad del proceso de integración, los gastos de constitución de la nueva entidad, de auditoría, de asesoramiento legal y empresarial, el alquiler de local y otros gastos de gestión.

3 Para un tratamiento más a fondo de la diversa patología de intercooperación empresarial cooperativas, Alfonso Sánchez, 2000, 37 y ss.

4 Embid, 1998, 11.

5 Alfonso Sánchez, 2000, 25. ISSN: 1134 - 993X, Núm. 44/2010, Bilbao, págs. 159-176 
dualidad propia, regulación peculiar en las leyes cooperativas y con una larga tradición en determinados sectores del cooperativismo ${ }^{6}$.

Estas son sociedades cooperativas compuestas por dos o más cooperativas (aunque se admite, por lo general, la participación como socios de otro tipo de entidades) que se unen con objeto de promover, coordinar y desarrollar fines económicos comunes de las entidades socias e integrar la actividad económica de las mismas. La flexibilidad que ofrece este peculiar subtipo social cooperativo permite alcanzar mayores o menores cuotas de integración dependiendo de la voluntad de sus miembros, que van desde facilitar meras colaboraciones empresariales hasta servir de marco organizativo a verdaderos fenómenos grupales a los que el legislador le da una específica vestidura jurídica?

Junto a las cooperativas de segundo grado, el otro gran instrumento de integración cooperativa lo constituyen los grupos cooperativos (art. 78 LCOOP), entes no personificados formados, en todo o en parte, por cooperativas que aunque formalmente autónomas, responden a las directrices fijadas por la entidad cabeza del grupo, y esta unidad de decisión, se traduce en una apariencia de unión económica entre las sociedades de base con la entidad que las dirige. Pero, al igual que pasaba con las cooperativas de segundo grado, el gran margen que se concede a la autorregulación normativa, en este caso a través del diverso contenido que se le puede dar, de haberlo, al contrato de organización del grupo en cuanto a los niveles de delegación de facultades a la dirección del grupo, permite alcanzar muy distintos niveles de integración empresarial.

También se debe englobar dentro de los fenómenos de integración cooperativa, ahora de ámbito internacional, el experimento, para potenciar la cooperación transfronteriza entre cooperativas, por ahora fracasado por su casi nula utilización práctica, de la Sociedad Cooperativa Europea (SCE).

Su régimen jurídico, contenido básicamente en su Estatuto aprobado por el Reglamento 1435/2003 (se cita RESCE) y completado en su ámbito laboral por Directiva 2003/72 del Consejo, no ha conten-

6 Por ejemplo, en el ámbito agrario en todo el territorio español se contabilizan actualmente 230 (datos de 2007 elaborados por CCAE, MAPA, 2007, 14). Aunque de las formulas de concentración e integración empresarial, tradicionalmente en España han sido las cooperativas de segundo grado las que han tenido una mayor implantación, en la última década, ante la insuficiencia demostrada por aquellas para alcanzar en determinados casos los umbrales suficientes de competitividad, se ha producido un notable avance en los procesos de fusión (sobre las ventajas e inconvenientes de las cooperativas de segundo grado frente a la fusiones de cooperativas: Meliá Martí, 2003, 110 y ss.).

7 Embid, 2004, 161. 
tado por muy diversos motivos (incertidumbre en el sistema de fuentes aplicables, modelo de cooperativa excesivamente economicista, complicaciones para su aplicación en España por la coexistencia de múltiples leyes cooperativas, problemas en cuanto a qué Registro es competente para su inscripción, etc.) a la numerosísima doctrina española que lo ha analizado ${ }^{8}$. Por otra parte, hasta ahora, de manera parecida a lo que ha ocurrido con la Sociedad Anónima Europea, los operadores económicos no han percibido a la SCE como un instrumento válido para abordar proyectos cooperativos de ámbito supranacional. Por ahora sólo diremos que la SCE puede constituirse ex novo, por personas físicas (mínimo cinco) y/o jurídicas (mínimo dos, por ejemplo cooperativas), mediante fusión de cooperativas o por la transformación de una cooperativa existente en SCE (art. 2.1 RESCE), siendo necesario, en todo caso, que todos los fundadores sean europeos o comunitarios y que, al menos, tengan vinculación con dos Estados miembros diferentes de la UE. El carácter de mecanismo de integración cooperativa se desprende claramente del RESCE cuando precisa que la SCE «tiene por objeto principal la satisfacción de las necesidades y el fomento de las actividades económicas y sociales de sus socios, en particular mediante la conclusión de acuerdos con ellos para el suministro de bienes o servicios, la ejecución de obras [...] o a través del fomento de su participación en actividades económicas en una o más SCE o sociedades cooperativas nacionales» (art. 1.3 RSCE).

Por debajo en la integración cooperativa, en cuanto a los niveles de ligazón empresarial, están las meras uniones de empresas, consorcios, agrupaciones de interés económico, conciertos, convenios, acuerdos y otras formas de colaboración económica intercooperativa (art. 79 LCoOp), que se utilizan para la realización conjunta de determinadas actividades económicas en interés común, normalmente de carácter complementario a la que desarrollan con carácter principal las cooperativas vinculadas, y normalmente sin gran vocación de permanencia.

\section{Integración y diferenciación cooperativa}

Conviene advertir, no obstante, que los problemas de tamaño de la cooperativa no se dan sólo por arriba, con el conocido mal endé-

8 Cobo del Rosal, 2003, 484.496; Martínez Segovia, 2003, 61-106; Pastor, 2001, 123-174, y 2008, 157-180; Vicent Chuliá, 2003, 51-83; Lambea, 2006, 85-112; Paniagua, 2007, 19-23 y un largo etcétera. 
mico del tejido empresarial español dominado de manera absoluta por PYMEs y microempresas, con una falta evidente de empresas fuertes y competitivas, sino que también se dan por abajo, puesto que un problema también clásico del cooperativismo es la carencia dentro de las propias sociedades cooperativas de mecanismos adecuados para la gestión empresarial de las particularidades y diferencias objetivas que puede haber en su seno, que muchas veces podrían explotarse, como de hecho suelen terminar haciéndose, con sociedades/empresas distintas. Y si se observa la realidad económica y para qué se utilizan los instrumentos jurídicos reseñados de concentración o integración empresarial, muchas veces su finalidad última no es crear una gran empresa o fortalecer la ya existente, sino simplemente permitir la subsistencia de cooperativas de reducida dimensión, que tendrían serias dificultades para competir en el mercado de manera aislada.

$Y$ es que los fenómenos de integración, como casi todo, dependen mucho desde el punto de vista del que se miren. Por ejemplo, ante un grupo de sociedades, si nos fijamos en la sociedad matriz, cabeza de grupo, nos puede dar la sensación de que estamos ante una gran empresa, conformada por el sumando de las filiales, y por ello las cuentas consolidadas que deben presentar dan la sensación numérica de una gran estructura corporativa. Pero si ahora nos fijamos en algunas de las sociedades filiales del mismo grupo, en concreto en las más pequeñas, que a pesar de esa dirección unificada son jurídicamente independiente de la sociedad matriz y con personalidad jurídica e identidad empresarial propia, se comprueba como el grupo es el instrumento jurídico-económico que se aprovecha, se supone que por el bien común, léase interés del grupo, de estas concretas empresas.

Y esto, si cabe, se percibe con mayor nitidez en los llamados grupos cooperativos paritarios como son los cooperativos, donde la sociedad cabeza de grupo o la cooperativa de segundo grado suele de menor tamaño, con menos entidad económica y con muchas menos capacidad operativa por lo limitado de la atribución de facultades por las sociedades de base, que la de la sumatoria de las múltiples entidades que las componen. En estos casos los grupos o las cooperativas de segundo grado son estructuras de red que sirven para compatibilizar las ventajas de las empresas que participan, permitiendo poner en común aquellos elementos de la actividad empresarial con respecto a los cuales pueden alcanzar sinergias significativas a la vez de mantener las ventajas de flexibilidad e implicación de cooperativas PYMEs $^{9}$.

9 Celaya/Arregui, 2005, 100. 
El mantenimiento de las diferencias internas de las cooperativas a veces se logra con la constitución de secciones cooperativas (art. 5 LCoop), peculiar instituto cooperativo con el que se pretende compensar la dificultad que tienen estas sociedades de contar con cooperativas filiales. Las secciones desarrollan dentro del objeto social de la cooperativa actividades económico-sociales específicas, y tienen autonomía de gestión, patrimonio separado y cuentas de explotación diferenciada, como si de unidades de negocio distinto se tratase.

Sin embargo no se pueden equiparar la autonomía patrimonial de las sociedades filiales respecto a su matriz con la de las secciones respecto a la sociedad cooperativa en la que se integran. Entre las sociedades filiales y entre éstas y la matriz, salvo casos excepcionales no opera una responsabilidad patrimonial conjunta o solidaria del grupo frente a terceros ${ }^{10}$. En cambio, en las cooperativas con secciones, aunque del cumplimiento de las obligaciones de la actividad de la sección responde, en primer lugar, las aportaciones hechas o prometidas por los socios integrados en las misma y, en su caso, el patrimonio afecto a las resultas de las operaciones que en su seno se realicen, al final, en caso de que aquéllas o ésta sean insuficientes, opera la responsabilidad universal de la cooperativa, con todo su patrimonio ${ }^{11}$.

De estos dos problemas relacionados con el tamaño ideal de la cooperativa (concentración/diferenciación), es, sin duda, el primero, con la constante búsqueda de caminos idóneos para lograr grandes estructuras empresariales a través de alguno de los distintos procedimientos económicos-jurídicos pensados para ello (fusión, cooperativas de segundo grado, grupos de sociedades) o de más simples e informales mecanismos de colaboración interempresarial (consorcios, uniones de empresas, agrupaciones, acuerdos intercooperativos, redes cooperativas), el que más ha preocupado al movimiento cooperativo, sin embargo no podemos obviar el segundo aspecto porque, como hemos visto, ambos están funcionalmente relacionados. Relación que también existe desde un punto de vista finalista, ya que ante ambos supuestos, necesidad de crecer o necesidad de diferenciación, si no se le da la adecuada respuesta a través de la articulación de mecanismos jurídicos eficaces el resultado final es el mismo: la siempre temida transformación o disolución de la cooperativa para constituir otro tipo de sociedad más flexible que faciliten la adecuación dimensional de la empresa a las demandas siempre cambiantes del mercado.

10 Girgado Perandones, 2002, 112 y ss.; Fuentes Naharro, 2007, 125 y ss.

11 Santos Martínez, 1982, 1071 y ss. 
Aunque la admite, el legislador cooperativo pone múltiples trabas a las operaciones de transformación de la cooperativa en otro tipo social, por ejemplo, con la obligación de devolver las subvenciones y ayudas fiscales recibidas por la cooperativa, con la pérdida de los fondos no repartibles (que van a parar a manos de la Administración que lo destina a determinadas fines relacionados con la promoción del cooperativismo: art. 69.6 LCoop) e incluso a veces, haciendo depender la operación de la pertinente autorización administrativa [art. 108.2, letra e) LCAND], algo esto último que nos parece fuera de lugar en el marco del Derecho Privado donde se deben enmarcan estas operaciones de reestructuración.

\section{Los grupos cooperativos y las limitaciones legales para la constitución de filiales}

Es un hecho cuantitativo que los grupos de empresas cooperativas ocupan un papel muy relevante en la economía española. El ejemplo más paradigmático lo encontramos con Mondragón Corporación Cooperativa (MCC), primer grupo empresarial del País Vasco y séptimo de España, que constituye un referente mundial del éxito empresarial del modelo cooperativo. En el sector financiero hay que señalar MAPFRE, Caja Laboral, CAJAMAR y las Cajas Rurales; en sector alimentario a GECV; en el del comercio minorista a CONSUM; y en el sector agroindustrial destacan, entre otros, ANECOOP, Agropecuaria de Guissona, COVAP, CASI, COREN, Agropecuaria de Navarra, Leyma, Hojiblanca, ACOR, ACOREZ, COPAGA, COPLACA, etc.

Pero una cosa son los grupos cooperativos propiamente dichos (compuestos sólo por cooperativas, aunque la cabeza de grupo no lo sea) y otra son los grupos de sociedades en los que participan una o varias cooperativas. Actualmente se ha superado el tradicional planteamiento endogámico en la conformación de grupos sólo por cooperativas y ya se admite la vinculación de cooperativas con sociedades de capital a través de la creación de sociedades filiales. En estos grupos heterogéneos, por las notas jurídicas que caracterizan a las cooperativas (un socio un voto, participación directa de los socios en el desarrollo de la actividad cooperativizada, etc.), estas sociedades suelen ocupar una posición jerárquicamente superior (en la cúspide de la pirámide, como sociedad matriz) a través de la participación mayoritaria de las cooperativas en una o varias sociedades de capital ${ }^{12}$.

12 Embid, 2003, 142 y s. 
Sin embargo, conviene apuntar que existen evidentes limitaciones para las cooperativas a la hora de constituir empresas filiales, participar en sociedades mercantiles, crear holding o constituir filiales comunes (joint ventures) con entidades mercantiles, ya que aunque todas las leyes cooperativas admiten en general dichas operaciones [art. 57.3, letra a) LCOoP; art. 134 LCPV, art. 129.2 LCCM, art. 102.1 LCCV, art. 160.1 LCAND], los beneficios generados por éstas no revierten directamente en los socios de la sociedad cooperativa matriz sino que deben destinarse a la dotación del FRO (aunque aquí se perciben algunas diferencia importantes entre las leyes de cooperativas, así, mientras la mayoría obliga a imputar el 100 por cien de dichos resultados al FRO, otras leyes, como la LCMur sólo obligan a dotar el 50 por cien). De otro lado, según el artículo 13.9 LRFC es causa de pérdida de la condición de cooperativa fiscalmente protegida, la participación de la cooperativa, en cuantía superior al 10 por cien en el capital social de entidades no cooperativas, porcentaje que se incrementa al 40 por cien cuando se trate de entidades que realicen actividades preparatorias, complementarias o subordinadas a las de la propia cooperativa. En todo caso, sigue diciendo la norma fiscal, el conjunto de estas participaciones en sociedades no mercantiles no podrá superar el 50 por cien de los recursos propios de la cooperativa, para lo que habrá que estar en la cuantía de los fondos sociales y del capital social no reembolsable.

En cuanto a los grupos cooperativos propiamente dichos, de la definición legal contenida en la LCoOP («conjunto formado por varias sociedades cooperativas, cualquiera que sea su clase y la entidad cabeza de grupo que ejercita las facultades o emite instrucciones de obligado cumplimiento para las cooperativas agrupadas» -art. 78.1-), se desprende la necesidad de una dirección económica unificada y no un mero control (unidad de decisión, que como señala también el artículo 4 LMV, es el elemento decisivo para individualizar el grupo desde un punto de vista legislativo). Esto es lógico si se tiene en cuenta la incompatibilidad de la existencia de grupos jerárquicos con los principios cooperativos que rigen en estas sociedades ${ }^{13}$, por lo que los grupos cooperativos a los que se refiere la LCoOp y las leyes autonómicas que los regulan (excepto la LCCAT que admite una composición heterogénea en el grupo —art. 125-) tienen que tener carácter de grupos paritarios o por coordinación.

En estos grupos paritarios no existe una relación de dependencia entre las sociedades agrupadas, de manera que la dirección unitaria

13 Embid, 1998, 14, y 2003, 151; Alfonso Sánchez, 2000, 147; en cambio lo admiten, por considerar que se pueden utilizar diversas formas contractuales de dominio por parte de una sociedad cooperativa respecto a otras, Aizega/Valiñani, 2003, 20 y s. 
no se impone por una entidad superior sino que la gestión del grupo se caracteriza por una coordinación horizontal a través de acuerdos de colaboración mutua entre las cooperativas miembros. De este modo, en los grupos paritarios la dirección unitaria es el elemento que aporta cohesión al grupo y en su determinación intervienen, en igualdad, todas las sociedades del grupo. La unidad de decisión, por tanto, recorre un camino inverso que en los jerárquicos o de subordinación, por lo que se pueden representar el grupo como una pirámide invertida en la que la cabeza de grupo se sitúa en una posición inferior y en la base, pero por arriba las sociedades que lo componen.

Podemos traer aquí el ejemplo del Grupo Caja Rural, cuya cabeza orgánica la ocupa la Asociación Española de Cajas Rurales, y a través de ella se articula la participación de las Cajas Rurales miembros (actualmente 73) en el Banco Cooperativo Español (BCE), Rural Grupo Asegurador (RGA) y Rural Servicios Informáticos (RSI). El sistema de integración utilizado por el grupo es un modelo de banca federada que permite salvaguardar la autonomía de las entidades asociadas que lo forman, sin tener que renunciar al requisito indispensable de operatividad bancaria y eficiencia empresarial, al tiempo que supera las limitaciones de cada entidad por su respectiva dimensión individual y el ámbito geográfico de su actividad. La fórmula de integración adoptado sigue los pasos de otros sistemas afines de banca cooperativa con larga tradición y éxito en Europa, algunos de los cuáles se clasifican entre los primeros grupos bancarios del continente, como son el Okobank finlandés, el Rabobank en los Países Bajos, el Österreichische Raiffeisenbanken austríaco, el Crédit Agricole en Francia o el sistema alemán Volksbanken-Raiffeisenbanken.

\section{Algunas experiencias empresariales para superar las limitaciones financieras que presenta el régimen de las sociedades cooperativas}

El régimen jurídico de las sociedades cooperativas presenta una serie de limitaciones para el desarrollo y expansión empresarial de estas sociedades. Aunque no es cierto que la formula cooperativa sólo sea apta para pequeñas empresas o para organizar una primera fase de explotación de una empresa, sí es un hecho constatado que cuando las cooperativas llegan a un determinado punto de desarrollo empresarial y alcanzan un volumen de negocio considerable muchas suelen terminar transformándose en otros tipos sociales para desarrollar así de manera más «eficiente» (teniendo en cuenta que la «eficiencia» está 
determinada precisamente por las leyes que rigen esas entidades) determinadas ramas de actividad y, de paso, sortear algunas de las limitaciones derivada de su peculiar estructura organizativa (dificultad para transmitir la posición de socio, límites de los derechos políticos y económicos de los socios inactivos, dificultad para la existencia de socios capitalistas), límites financieros (dificultad de concentrar capital social y atraer inversión externa, obligación de dotación de fondos de carácter irrepartible), y limites operativos o funcionales (límites a las operaciones con terceros, límites para invertir en sociedades mercantiles) ${ }^{14}$.

Deberíamos meditar sobre todo esto y preguntarnos por qué muchas cooperativas han acudido a verdaderas operaciones de ingeniera societaria para sortear los obstáculos que para su crecimiento y expansión encontraban en la legislación cooperativa.

En España, un buen ejemplo de lo que decimos lo tenemos en el caso del Grupo Alimentario de Guissona ${ }^{15}$. En 1999, la dirección de la Cooperativa Agropecuaria de Guissona, ante la insuficiencia del modelo cooperativo decidió constituir una SA a la que cedió gran parte de sus activos y ramas de actividad. El precedente fue la creación durantes los años 90 de una serie de sociedades mercantiles como filiales de la cooperativa, que desarrollaban distintas líneas de negocio y que constituyeron nuevas líneas de actuación estratégica, en la medida que ampliaban las posibilidades comerciales de la empresa. La Corporación Alimentaria Guissona SA se constituyó con las aportaciones no dinerarias de cinco sociedades participantes (la cooperativa y cuatro filiales de ésta), aglutinando toda la actividad comercial, industrial y los servicios técnicos del grupo. En concreto, la cooperativa aportó varias ramas de actividad; tres filiales (Area de Guissona SA, Propor 2000 SA y Alpisa SA) y la totalidad de sus ramas de actividad, además de tres explotaciones porcinas en pleno funcionamiento (Alsicor SA). Sin embargo, un grupo de socios de la CAG, respaldados por el sindicato Unió de Pagesos y la Federación de Cooperativas Agrarias de Cataluña, impugnó la reorganización empresarial al considerar que con el trasvase de más del 70 por cien de la actividad a Corporación Alimentaria se vulneró el régimen legal de las cooperativas y se infravaloraron los activos de la entidad. El juzgado de Cervera desestimó la demanda, y el caso llegó a la Audiencia de Lleida, que ratificó la viabilidad de la operación. Posteriormente la cooperativa ofreció a sus socios la adquisición de parte de las acciones de la Corporación. El incremento del valor de las acciones

14 Con más detalle, Vargas Vasserot, 2008, passim.

15 Bien relatado en Roca i Trescent/Roca i Puigvert, 2004. 
ha sido constante y se han planteado incluso su transformación en sociedad cotizada. En la actualidad la sociedad está controlada por los socios de la cooperativa, dado que la sociedad matriz paulatinamente ha ido vendiendo sus acciones a sus propios socios.

Otro ejemplo lo tenemos en COREN (Cooperativa Ourensana), cooperativa de segundo grado conformada por 15 cooperativas de primer grado de cría de ganado, que constituye el primer grupo alimentario gallego y el mayor grupo cooperativo agroalimentario español. Desde los años 90 ha afrontado un espectacular proceso de expansión a través de creación y adquisición de sociedades mercantiles (anónimas y limitadas) como filiales, que utiliza para la distribución, transformación y comercialización de sus productos, constituyendo también sociedades mercantiles en el extranjero, y explotando una red de franquicias con tiendas y restaurantes. En su seno ha habido varios procesos de constitución y adquisición de sociedades anónimas para explotar ramas de actividades complementarias a la principal de las cooperativas socias, y para ello algunas de las cooperativas de primer grado han tenido que aportar determinados activos y rama de actividad para la constitución de filiales del grupo.

Fuera de España también se han producido fenómenos parecidos, que aunque sea brevemente reseñaremos en sus aspectos más significativos, señalando, no obstante, la existencia de régimenes legales más permisivos con los procesos de expansión y diversificación empresarial.

En Irlanda la cooperativa lechera Kerry Co-op se constituyó en 1974 y en 1986, ante la necesidad de expansión y de integración de procesos de comercialización, creó una sociedad anónima (Kerry Group Public Limited) con dos tipos de acciones, unas que cotizaban en bolsa y transmisibles (tipo A) y otras no transmisibles (tipo B). Estas últimas, que suponían el 60\% del capital social de la SA, fueron entregadas a la cooperativa como pago por la aportación de determinadas ramas de actividad (transformación y comercialización de leche), de activos y de varias sociedades filiales. Las acciones de tipo A fueron vendidas a los socios y empleados de la cooperativa, con lo que la mayoría de ellos se hicieron accionistas directos de la nueva sociedad, siendo el resto ofrecida a inversores externos en la Bolsa de Dublín. La cooperativa sigue funcionando con su actividad original y los socios activos siguen realizando la actividad cooperativizada típica (aportan leche). La estructura híbrida original de la SA constituida (acciones tipo A y acciones tipo B) permitía el control por parte de la cooperativa, que siguió siendo durante años el accionista mayoritario. Los importantes dividendos que recibe la cooperativa por la buena marcha de la SA se distribuyen entre sus socios en forma de retornos. Por su parte, los socios titulares de acciones de la SA además de recibir dividendos directos de la sociedad, 
siempre tienen la posibilidad de obtener beneficios con la venta de sus acciones en el mercado secundario. Desde la constitución de la SA el crecimiento empresarial del grupo ha sido espectacular, con una continua diversificación de las líneas de negocio y desarrollo de nuevos productos. Hoy día, la producción láctea del grupo, que fue el origen de su actividad, representa sólo el 10 por cien de su cifra de negocio, contando con sociedades filiales en más de 20 países, constituyendo las ventas de productos fuera de Irlanda más del 80 por cien del total. En la actualidad, la estructura accionarial de la SA ha cambiado, ya que una buena parte de las acciones de tipo B, pertenecientes a la cooperativa, se han convertido en acciones transmisibles de tipo A con el objeto de financiar determinados proyectos de expansión que la cooperativa quiere afrontar. Con ello la cooperativa ha disminuido su participación en la SA, perdiendo su control mayoritario. No obstante, los socios son aún poseedores del 20 por cien de las acciones de tipo A, con lo que todavía la cooperativa y sus socios controlan la SA.

En Holanda, la cooperativa lechera Campina se creó en 1989 por la fusión de dos importantes cooperativas lecheras de los países bajos. La cooperativa resultante constituyó con la aportación de determinadas ramas de actividad una SA (Campina BV), especializada en el transformación, venta y distribución de productos de consumo lácteos y derivados de lácteos e ingredientes del catering y la farmacia. A diferencia del caso anterior, los socios cooperativistas no son accionistas directos de la SA, por lo que los socios se benefician de la buena marcha de la sociedad mercantil por el incremento en el precio de la actividad cooperativizada que realizan y porque los retornos se articulan mediante la entrega de obligaciones subordinadas, reembolsables, retribuidas (interés superior al 5 por cien) y negociables, que pueden ser vendidas a la cooperativa de manera anticipada y a un precio menor del nominal. El 2 de junio de 2006, la Comisión Europea decidió no oponerse a la alianza empresarial suscrita entre esta sociedad y el grupo cooperativo neozelandés Fonterra, para la creación de una gran sociedad dedicada a la venta de lactosa (2006/C 194/09).

En Canadá, Saskatchewan Wheat Pool (SWP), creada en 1924, constituye una de las más importantes empresas de venta de cereal del país, siendo la cooperativa agroalimentaria más importantes. (Tras la compra de su competidora, la empresa de Winnipeg Agricore United, SWP cambió su nombre por Viterra). En 1994 la cooperativa se encontró con un grave problema social, ya que el 46 por cien de sus miembros tenían una edad superior a 55 años y poseían un 30 por cien del capital social, capital que le tenía que ser reembolsado a los socios cuando cumpliesen los 70 años por el sistema de capital rotativo dis- 
puesto en sus estatutos. Además, la cooperativa consideró que era necesario realizar una importante política de inversión para modernizar sus instalaciones, por lo que decidió convertirse en una cooperativa por acciones, opción reconocida en la legislación canadiense. Esto obligó a reconvertir todas las participaciones sociales de la cooperativa en acciones de clase A (con derecho a voto) y de clase B (sin derecho a voto). Las primera fueron vendidas a los productores socios de la cooperativa; mientras que las acciones de tipo $B$, sin derecho a voto, que estaban destinadas a inversores externos, se ofrecieron primero a los socios y empleados de la cooperativa a través de un programa para la adquisición de las mismas. El sistema para la atribución de acciones se hizo de la siguiente manera. Las 25 primeras participaciones de cada socio fueron convertidas en acciones de clase A (se crearon 76.600 acciones). El resto de participaciones de cada socio fueron transformadas en acciones de tipo $B$ en un ratio de 10 por 1 (se crearon 30.344 .800 acciones), que podían venderse a inversores externos mediante su cotización en bolsa. No obstante, para evitar el control de la cooperativa por un pequeño grupo de socios, se estableció un límite de 10 por cien de acciones de clase $\mathrm{B}$ por cada socio.

En Estados Unidos, Dakota Growers Pasta Company era una de las cooperativas agrícolas más importantes de los Estados Unidos, dedicada a la transformación de pasta. En los años noventa inicia un proceso de expansión a través de la adquisición de una serie de sociedades mercantiles. En 2002, y ante las necesidades de obtener inversión externa para afrontar nuevos proyectos empresariales, la sociedad decide abandonar el modelo cooperativo al considerar que éste limitaba sus posibilidades de expansión y de penetración en nuevos mercados y no permitía la obtención de recursos externos. La cooperativa matriz se transformó en una sociedad anónima, creándose distintos tipos de acciones. A los socios de la cooperativa se le da la opción de convertirse en accionistas de la SA, y si no lo hacían tenían la posibilidad de seguir aportando sus productos a la nueva sociedad. Aunque la sociedad consiguió solventar a corto plazo sus problemas de financiación, por el camino se perdió la identidad cooperativa cuando finalmente el control de la sociedad fue tomado por socios no cooperadores.

\section{Conclusiones}

Hay que crear mecanismos para que las grandes cooperativas, a pesar de la atracción que genera modelos más capitalistas, quieran, 
voluntariamente mantenerse en el modelo cooperativo, con todas las ventajas que conllevan las empresas de Economía Social para los socios, los trabajadores y, en general, para la sociedad. Las grandes cooperativas de nuestro país son el motor de desarrollo de determinadas zonas territoriales por lo que su hipotética desaparición produciría una gran convulsión social que repercutiría negativamente en la imagen de la sociedad y en la de sus dirigentes. Esa pérdida sería traumática para un buen número de socios, que considerarían inaceptable que la cooperativa de su localidad, orgullo de todos, se convierte en una sociedad mercantil, con el riesgo de deslocalización que eso siempre conlleva.

Pero si ante la maliciosa pregunta de ¿cuántas sociedades anónimas se han transformado en cooperativas en nuestro España?, la respuesta es que muy pocas, por no decir ninguna, es que algo falla. Habrá que cambiarlo, y mientras el legislador reacciona buscar respuestas imaginativas, que las hay (constitución de filiales, cesión de activo y pasivo, configurar correctamente el margen de autorregulación estatutaria, creación de secciones, etc.) para que los socios no vean en el tipo cooperativo una rémora a sus intereses.

Así, a pesar del entorno legal "pro» cooperativas que existe en España, en particular, potenciando los procesos de integración, la legislación específica sobre esta materia no es la adecuada. Como hemos podido comprobar, la legislación contiene normas que, en cierta medida, limitan las posibilidades de crecimiento y expansión en el modelo cooperativo, lo que ha provocado la búsqueda de alternativas de auténtica ingeniería societarias para eludir tales limitaciones e incluso la transformación de la cooperativa en otro tipo de sociedad mercantiles, digámoslo así, más cómodas para diversificar, crecer y competir en el mercado. Por ello, consideramos que el régimen legal necesita reconocer las posibilidades de crecimiento y diferenciación, permitiendo flexibilidad para que las cooperativas crezcan de tamaño sin perder de vista los principios cooperativos, que, eso sí, deben ser reinterpretados según los nuevos tiempos que corren.

\section{Bibliografía}

Alfonso Sánchez, R., Integración cooperativa y sus técnicas de realización: la Cooperativa de Segundo Grado, Valencia, Tirant Lo Blanch, 2000.

Barea, J., Julía, J. Fco. y Monzón, J.L., Grupos empresariales de la Economía Social en España, CIRIEC, Valencia, 1999. 
Celaya, A. y Arregui, Z., "Bases para la reforma de la legislación cooperativas europea», Boletín de la Academia Vasca de Derecho, núm. 8, 2005, pp. 95-106.

ChAVES/FAJARDO/NAMORADO, Integración empresarial cooperativa: posibilidades, ventajas e inconvenientes, Ciriec-España, Valencia, 2003.

CoBo del Rosal, A., «Principales aspectos del nuevo régimen jurídico de la Sociedad Cooperativa Europea (Reglamento núm. 1435/2003, del Consejo Europeo, de 22 de julio de 2003)», RdS, núm. 21, 2003, pp. 484-496.

Embid Irujo, A., Concentración de empresas y Derecho de cooperativas, Murcia, Universidad de Murcia, 1991.

EMBID IRUjo, A., «Problemas actuales de la integración cooperativa», RDM, núm. 227, 1988, pp. 7-36.

Fuentes NaharRo, M., Grupos de sociedades y protección de acreedores (una perspectiva societaria), Madrid, Civitas-Aranzadi, 2007.

Giagnocavo, C., «Legislative Change in the Agricultural Cooperative Sector: Imitation, Evolution or Innovation?», Innovation and Management: Answers to the great challenges of public, social economy and cooperative enterprises, CIRIEC, Seville, 2008.

GiRgado Perandones, P., La responsabilidad de la sociedad matriz y de los administradores en una empresa de grupo, Madrid, Marcial Pons, 2002.

Lambea Rueda, A., "Marco jurídico de la Sociedad Cooperativa Europea domiciliada en España», CIRIEC-Jurídica, núm. 17, 2006, pp. 85-111.

Martínez Segovia, F.J., «Primera aproximación al Estatuto de la Sociedad Cooperativas Europea», REVESCO, núm. 80, 2003, pp. 89 y ss.

Melía Martí, E., Juliá Igual, J. y Martínez García, A., "Los procesos de fusión de cooperativas en España y su incidencia en la competitividad: Un estudio empírico», Actas del XXVII Congreso Internacional CIRIEC, 2008 (publicación digital).

PAniagua, M., «El estatuto de la sociedad cooperativa Europea: el problema de su aplicación en España», La Sociedad Cooperativa, núm. 34, 2007, pp. 19-23.

PAstor Sempere, C., «La Sociedad Cooperativa Europea: la compleja articulación de un nuevo tipo societario», en Internacionalización de las cooperativas, MorÁn García (coord.), Alicante, Universidad de Alicante, 2008, pp. 157180.

PAstor Sempere, C., «La sociedad cooperativa europea», REVESCO, núm. 74, 2001, pp. 123-174.

RocA I TRESCENT, J. y RocA I PUIGVERT, M.R., Desarrollo y gestión de una transformación empresarial: Grup Alimentari de Guissona, Lleida, Universitat Pompeu Fabra, 2004, disponible en http://www.econ.upf.edu/docs/case_studies/ 44.pdf.

Santos Martínez, V., "Las secciones de las cooperativas en Derecho español», en Estudios de Derecho Mercantil en homenaje al profesor Antonio Polo, Barcelona, 1982, pp. 1071-1140.

VARGAS-VASSEROT, C., "Limitaciones del modelo cooperativo y necesidad de reforma de su régimen legal», Economía Social, n. 46, 2008, pp. 10-18. 
VARGAS-VASSEROT, C., «La actividad cooperativizada y las relaciones de la cooperativa con sus socios y con terceros», RdS Monografía n. 27, Pamplona, Thomson-Aranzadi, 2006.

VARgas-VASSEROt, C., Régimen jurídico de la Sociedad Cooperativa del siglo XXI. Realidades y propuestas de reforma, Gadea/Sacristán/Vargas Vasserot, Dykinson, 2009, pp. 535-544.

VICENT Chulí, F., "La Sociedad Cooperativa Europea», CIRIEC-Jurídica, núm. 14, pp. $51-82$. 